\title{
openheart Pre-test characteristics of unstable angina patients with obstructive coronary artery disease confirmed by coronary angiography
}

\author{
Kristina Fladseth, ${ }^{1,2}$ Andreas Kristensen, ${ }^{2}$ Jan Mannsverk, ${ }^{2}$ Thor Trovik, ${ }^{2}$ \\ Henrik Schirmer ${ }^{3,4}$
}

\begin{abstract}
- Additional material is published online only. To view please visit the journal online (http://dx.doi.org/10.1136/ openhrt-2018-000888).
\end{abstract}

To cite: Fladseth $\mathrm{K}$ Kristensen A, Mannsverk J, et al. Pre-test characteristics of unstable angina patients with obstructive coronary artery disease confirmed by coronary angiography. Open Heart 2018;5:e000888. doi:10.1136/ openhrt-2018-000888

Received 25 June 2018 Revised 10 October 2018 Accepted 17 0ctober 2018

A Check for updates

(C) Author(s) (or their employer(s)) 2018. Re-use permitted under CC BY-NC. No commercial re-use. See rights and permissions. Published by BMJ.

${ }^{1}$ Department of Clinical Medicine, UiT The Arctic University of Norway, Tromsø, Norway

${ }^{2}$ Division of Cardiothoracic and Respiratory Medicine, University Hospital of North Norway, Troms $\emptyset$, Norway ${ }^{3}$ Institute of Clinical Medicine, University of Oslo, Oslo, Norway ${ }^{4}$ Department of Cardiology, Akershus University Hospital, Lørenskog, Norway

Correspondence to Dr Kristina Fladseth; kristina. fladseth@unn.no

\section{ABSTRACT}

Objective Patients referred for acute coronary angiography (CAG) with unstable angina (UA) have low mortality and low rate of obstructive coronary artery disease (CAD). Better pre-test selection criteria are warranted. We aimed to assess the current guidelines against other clinical variables as predictors of obstructive CAD in patients with UA referred for acute CAG.

Methods From 2005 to 2012, all CAGs performed at the University Hospital of North Norway, the sole provider of $C A G$ in the region, were recorded in a registry. We included 979 admissions of UA and retrospectively collected data regarding presenting clinical parameters from patient hospital records. Obstructive CAD was defined as $\geq 50 \%$ stenosis and considered prognostically significant if found in the left main stem, proximal LAD or all three main coronary arteries. Characteristics were analysed by logistic regression analysis. A score was developed using ORs from significant factors in a multivariable model.

Results The overall rate of obstructive CAD was $45 \%$, and the rate of prognostically significant CAD was $11 \%$. The risk criteria recommended in American College of Cardiology/American Heart Association and European Society of Cardiology guidelines had an area under the curve (AUC) of 0.58 . Adding clinical information increased the AUC to 0.77 (95\% Cl 0.74 to 0.80$)$. Applying the derived score, we found that $56 \%(n=546)$ of patients had a score of $<13$, which was associated with a negative predictive value of $95 \%$ for prognostic significant CAD. Conclusions The current results suggest that CAG may be postponed or cancelled in more than half of patients with UA by improving pre-test selection criteria with the addition of clinical parameters to current guidelines.

\section{INTRODUCTION}

Acute chest pain is one of the most common presenting symptoms in emergency departments. ${ }^{1}$ It poses a challenge to healthcare systems as critical conditions require prompt diagnosis and treatment, whereas benign disorders need to be identified early to prevent unnecessary and potentially harmful procedures. Suspected acute coronary syndrome refers to patients with chest

\section{Key questions}

What is already known about this subject?

- Patients with unstable angina have a low mortality and a low rate of obstructive coronary artery disease.

- Applying symptom characteristics to traditional risk factors improves risk prediction models in patients with stable angina.

What does this study add?

- This study demonstrates that by structuring symptom characteristics and clinical variables it is possible to improve pre-test selection beyond guidelines risk criteria.

How might this impact on clinical practice?

- Better pre-test selection criteria for acute coronary angiography in patients with unstable angina would reduce cost for healthcare systems and avoid exposing patients to unnecessary risk of complications.

- Prospective studies are needed to validate our findings.

pain presumably caused by acute myocardial ischaemia and encompasses myocardial infarction (MI) and unstable angina (UA). Patients with UA have no evidence of myocardial injury. ${ }^{2}{ }^{3}$ New, high-sensitive cardiac troponin (hs-cTn) assays detect myocardial injury in a group of patients previously diagnosed as UA, thus changing the diagnosis to MI. ${ }^{4-6}$ Consequently, the present UA population have lower mortality and are less likely to have obstructive coronary artery disease (CAD)..$^{78}$

Despite this, the fear of missing an impending MI results in a liberal referral practice of patients with presumed UA to acute coronary angiography (CAG). European Society of Cardiology (ESC) and American College of Cardiology/American Heart Association (ACC/AHA) guidelines for the management of acute coronary syndrome in 
patients without ST elevation recommend performing CAG within 72 hours if there is either an intermediate Global Registry of Acute Coronary Events (GRACE) risk score (109-140), relevant comorbidity, recurrence of symptoms or a positive ECG or stress test. ${ }^{39}$ GRACE risk score predicts the risk of MI and death and is included in the guidelines as a tool to risk stratify these patients. ${ }^{10}$

A better pre-test selection is warranted. We aimed to assess the GRACE risk score, guidelines risk criteria and other clinical factors capability of predicting obstructive CAD in patients referred for acute CAG on the indication of UA.

\section{METHODS \\ Study population}

Between 1 January 2005 and 31 December 2012, all coronary angiographies (CAG) performed at the University Hospital of North Norway were recorded in a clinical registry. The University Hospital is the sole provider of CAG in Northern Norway, serving a local population of 127000 and a total regional population of 481000 . We included the 1936 CAGs performed in patients with presumed UA from the local catchment area to facilitate further retrospective data collection from patient hospital records. Patients with more than one procedure per admission were only included once $(n=35)$, and patients with a peak troponin level above the 99th percentile $(n=813)$ were excluded. ${ }^{2}$ We also excluded patients mislabelled as UA $(n=46)$ and patients with other primary local hospitals $(n=30)$, incorrectly registered as local patients. Patients who had undergone percutaneous coronary intervention (PCI) within the last 30 days $(n=33)$ were excluded because $91 \%$ of these patients had obstructive CAD, warranting acute CAG. Subsequently, the final cohort included 979 UA patient admissions.

\section{Data collection}

The registry contains data from all consecutive CAGs, recorded by the operator at the time of the procedure. Linkage to troponin levels from the Department of Clinical Chemistry at the University Hospital of North Norway and to patient hospital records was done by the national 11-digit identification number. From patient hospital records, we retrospectively collected data on symptoms and clinical findings at presentation, preceding symptoms, stress tests, risk factors, comorbidities and medication. The extent of CAD was evaluated by the interventional cardiologist. In patients with prior coronary artery bypass grafting, only those with new obstructive CAD were labelled with obstructive CAD. From July 2009, hs-cTnT replaced standard troponin assay. A standard troponin value of $10 \mathrm{ng} / \mathrm{L}$ corresponds to $30 \mathrm{ng} / \mathrm{L}$ hs-cTnT. To adjust for this, the troponin values measured up to July 2009 were multiplied by a factor of three. ${ }^{11}{ }^{12}$ In addition, we performed sensitivity analyses on the subpopulation with measured hs-cTnT.
Patients were referred as UA if chest pain at rest, new-onset angina or rapidly worsening angina. We registered the threshold of angina prior to admission by the Canadian Cardiovascular Society grading of angina pectoris. A variation in the threshold of angina of two or more grades was defined as a variable threshold. A declining threshold of angina was not included as a variable threshold. Refractory angina was recorded if intravenous nitroglycerine was given. We defined a history of typical angina as (1) substernal chest pain or discomfort, (2) provoked by physical exertion or emotional stress and (3) relieved by rest within minutes. Atypical angina was defined as two of these characteristics, and patients with one of these characteristics were defined as having non-anginal chest pain. ${ }^{13}$ A positive stress ECG was defined as $\geq 1 \mathrm{~mm}$ of ST-segment depression or elevation, or stress-induced chest pain. The guideline criterion of acute heart failure was defined as Killip class II-IV. We calculated the GRACE risk score according to the Fox model for death between hospital admission and 6 months (http://www.outcomes-umassmed.org/grace/ files/GRACE_RiskModel_Coefficients.pdf). $\quad$ Family history of CAD was defined as first-degree relatives with premature CAD stated in the patient hospital record. Diabetes mellitus was defined if the diagnosis occurred in the patient hospital records or HbAlc $\geq 6.5 \%$. Hypercholesterolaemia was defined by the use of lipid-lowering drugs or serum cholesterol level of $\geq 6.5 \mathrm{mmol} / \mathrm{L}$.

\section{Endpoint}

As the mortality is very low in patients with UA in the hs-cTn era, we chose obstructive CAD as the primary endpoint of our analyses. To ensure high sensitivity, obstructive CAD was defined as $\geq 50 \%$ angiographic diameter stenosis or fraction flow reserve $<0.8$ in any epicardial coronary artery. ${ }^{14}$ We defined obstructive CAD in the main stem, proximal left anterior descending artery or in all three main coronary vessels (three-vessel disease) as prognostically significant CAD. ${ }^{14}{ }^{15}$ UA resembles stable angina, both having negative hs-cTn and low mortality compared with MI, and an unsure prognostic benefit of revascularisation. Therefore, we assumed no immediate yield of acute revascularisation in the hs-cTn-negative UA patients without prognostically significant CAD.

\section{Statistical analysis}

Patient characteristics were reported as counts, percentages or means $\pm \mathrm{SD}$. Logistic regression analysis was used to investigate predictors of obstructive $\mathrm{CAD}$. In the final multivariable model, we included the predictors with clinical significance and $p<0.05$. We included interaction terms significantly improving the model by receiver operating characteristics (ROC) and the Net Reclassification Improvement. The Hosmer-Lemeshow goodness-of-fit test was not significant for the final model. To investigate the main contributing variables of the GRACE risk score and guidelines risk criteria, we used a forward selection logistic regression analysis, with inclusion at $\mathrm{p}<0.05$. 
We found that an increasing number of variables with missing information was significantly associated with no obstructive CAD (odds ratio (OR) $0.77,95 \%$ confidence interval (CI) 0.71 to 0.83 ). We tested this assumption for all variables included in the final model; it was found to be true for all variables except symptom characteristics. Therefore, missing information was combined in the reference group for the other variables, but classified as an independent predictive category for symptom characteristics.

We created a score based on the final multivariable model, weighting the variables with the OR rounded off to the nearest integer. Applying the score, we estimated the proportion of patients with a high negative predictive value $(\mathrm{NPV})$ for prognostically significant $\mathrm{CAD}$, assuming these patients could have been safely discharged without a CAG or referred for elective CAG. The discriminative performance of the GRACE risk score, the ESC and ACC/ AHA guidelines risk criteria, and the derived model and its score were tested by ROC analysis. Statistical analyses were performed with Stata V.14.0. All reported differences had two-sided $\mathrm{p}$ values $<0.05$.

\section{RESULTS}

\section{Patient characteristics}

Of the 979 patients with UA, the overall rate of obstructive CAD was $45 \%(n=443)$, falling from $56 \%(n=70)$ in 2005 to $29 \%$ ( $\mathrm{n}=33$ ) in 2012 ( $\mathrm{p}$ for trend $<0.001$ ). Obstructive
CAD of prognostic significance was prevalent in $11 \%$ $(n=103)$ of the patients. Patient characteristics are shown in table 1. Patients with obstructive CAD were older, more often male, smoked more, had more hypertension and hypercholesterolaemia, a higher GRACE risk score and a higher rate of established CAD.

\section{Performance of GRACE risk score and risk criteria from guidelines}

We found that both patients with and without obstructive CAD had low GRACE risk scores, 83 versus 76, respectively. In total, $<1 \%(\mathrm{n}=7)$ of the patients with UA had a high GRACE risk score $(>140)$ and $11 \%(\mathrm{n}=104)$ had an intermediate GRACE risk score (109-140). In patients with a high GRACE score, five out of seven patients had obstructive CAD versus half of the patients with an intermediate GRACE score. According to the ESC guidelines, $21 \%(n=202)$ of the patients in our study were candidates for a selective invasive strategy based on the results of a non-invasive stress test. However, 26\% ( $n=52)$ of these patients had obstructive $\mathrm{CAD}$ and $5.5 \%(\mathrm{n}=11)$ had prognostic significant CAD. ACC/AHA guidelines would allocate conservative treatment to $31 \%(n=299)$ of the patients, of which $32 \%$ $(\mathrm{n}=96)$ had obstructive CAD and $4.3 \%(\mathrm{n}=13)$ prognostic significant CAD. High-risk criteria from ESC and ACC/AHA guidelines were present in $25 \%(\mathrm{n}=242)$ and $22 \%(\mathrm{n}=216)$ of the patients, respectively. These

\section{Table 1 Patient characteristics}

\begin{tabular}{|lllc|}
\hline & $\begin{array}{l}\text { Obstructive CAD } \\
(\mathbf{n = 4 4 3 )}\end{array}$ & $\begin{array}{l}\text { No obstructive CAD } \\
(\mathbf{n = 5 3 6})\end{array}$ & P values \\
\hline Age (years) & $65 \pm 11$ & $60 \pm 12$ & $<0.001$ \\
\hline Male gender $(\%, \mathrm{n})$ & $67 \%(297)$ & $52 \%(281)$ & $<0.001$ \\
\hline BMI $\left(\mathrm{kg} / \mathrm{m}^{2}\right)$ & $28 \pm 5$ & $28 \pm 6$ & 0.543 \\
\hline Heart rate (beats/min) & $68 \pm 14$ & $71 \pm 16$ & 0.014 \\
\hline Systolic blood pressure $(\mathrm{mm} \mathrm{Hg})$ & $145 \pm 22$ & $140 \pm 21$ & $<0.001$ \\
\hline Diastolic blood pressure $(\mathrm{mm} \mathrm{Hg})$ & $81 \pm 12$ & $80 \pm 13$ & 0.223 \\
\hline Use of antihypertensive drugs $(\%, \mathrm{n})$ & $77 \%(339)$ & $63 \%(339)$ & $<0.001$ \\
\hline Hypercholesterolaemia $(\%, \mathrm{n})$ & $74 \%(326)$ & $66 \%(352)$ & 0.008 \\
\hline Diabetes mellitus $(\%, \mathrm{n})$ & $18 \%(79)$ & $15 \%(82)$ & 0.287 \\
\hline Established coronary artery disease $(\%, \mathrm{n})$ & $59 \%(263)$ & $39 \%(209)$ & $<0.001$ \\
\hline Previous MI $(\%, \mathrm{n})$ & $36 \%(158)$ & $21 \%(113)$ & $<0.001$ \\
\hline Previous PCl $(\%, \mathrm{n})$ & $46 \%(205)$ & $33 \%(177)$ & $<0.001$ \\
\hline Previous CABG $(\%, \mathrm{n})$ & $18 \%(80)$ & $14 \%(74)$ & 0.069 \\
\hline Family history of CAD $(\%, \mathrm{n})$ & $50 \%(220)$ & $53 \%(285)$ & 0.274 \\
\hline Smoking status & & & 0.008 \\
\hline Current smoker $(\%, \mathrm{n})$ & $29 \%(130)$ & $27 \%(143)$ & \\
\hline Former smoker $(\%, \mathrm{n})$ & $44 \%(195)$ & $38 \%(201)$ & $<0.001$ \\
\hline GRACE risk score & $83 \pm 22$ & $76 \pm 24$ & \\
\hline
\end{tabular}

Values are $\%(n)$ or mean \pm SD.

BMI, body mass index; CABG, coronary artery bypass grafting; CAD, coronary artery disease; GRACE, Global Registry of Acute Coronary Events; MI, myocardial infarction; $\mathrm{PCI}$, percutaneous coronary intervention. 


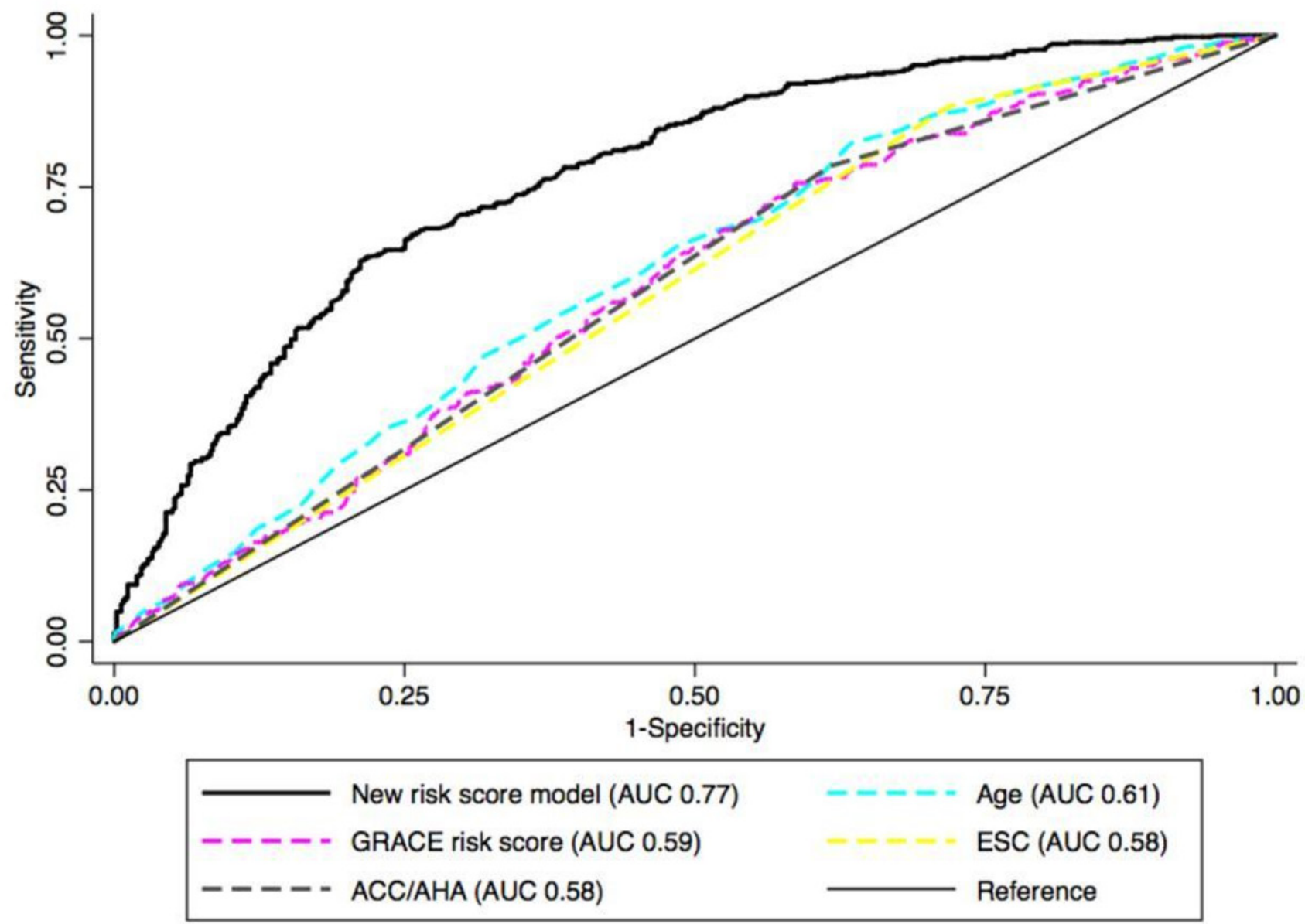

Figure 1 Prediction of obstructive coronary artery disease in unstable angina patients referred for coronary angiography. Receiver operating characteristics curves for age, Global Registry of Acute Coronary Events (GRACE) risk score, European Society of Cardiology (ESC) and American College of Cardiology/American Heart Association (ACC/AHA) guidelines risk criteria, and the new risk score model. AUC, area under the curve.

patients did not have more CAD than patients with intermediate-risk criteria.

GRACE risk score, ESC and ACC/AHA guidelines had similar area under the curve (AUC) for obstructive CAD, with AUC of 0.59 (95\% CI 0.55 to 0.62$), 0.58$ (95\% CI 0.56 to 0.61 ) and 0.58 (95\% CI 0.55 to 0.61 ), respectively. Age alone had a significantly higher AUC of 0.61 (95\% CI 0.58 to $0.65, \mathrm{p}=0.037$ ) (figure 1 ). The main contributing variables in the GRACE risk score and guidelines risk criteria were age, systolic blood pressure at admission, prior PCI, Killip class and a positive stress test. We did not find more ST-T abnormalities in the ECG of patients with obstructive CAD.

\section{Prediction of obstructive coronary artery disease}

A history of typical angina symptoms, Canadian Cardiovascular Society angina grade 3 or 4, no variable threshold of exertional angina, no history of palpitations, prior PCI, positive stress testing, smoking, hypertension, age $>65$ years and male gender all added independently significant information in a multivariable model, increasing the AUC for obstructive CAD to 0.77 (95\% CI 0.74 to 0.80 , $\mathrm{p}<0.001$ ) (table 2, figure 1), significantly higher than the GRACE risk score and guidelines risk criteria. The significant interaction term between age and prior PCI was also included. From the model, we derived a score predicting obstructive CAD with an OR of 1.40 (95\% CI 1.33 to 1.47, $\mathrm{p}<0.001)$ per score level increase. With a cut-off level of $<13$, the NPV was $95 \%$ for prognostic significant CAD in $56 \%(\mathrm{n}=546)$ of patients with UA referred for acute CAG. For the $44 \%(n=295)$ of patients with a score $<12$ the NPV was $97 \%$. Stratified by sex, a cut-off level of $<14$ gave a negative predictive value of $95 \%$ for $82 \%$ ( $n=330$ ) of females, and a cut-off level of $<12$ and $<13$ gave NPVs of respectively $96 \%$ for $20 \%(\mathrm{n}=177)$ and $93 \%$ for $43 \%$ $(\mathrm{n}=251)$ of males (table 3$)$.

In univariable analysis, shorter pain duration predicted obstructive CAD ( $<2-6$ hours, OR $1.82,95 \%$ CI 1.34 to $2.48, \mathrm{p}<0.001)$, whereas chest pain related to change in body posture $(\mathrm{n}=23)$ gave lower odds for obstructive CAD (OR 0.18, 95\% CI 0.05 to 0.68 ). We found that pain relief by nitrates, dyspnoea, pain radiation and number of chest pain episodes during the last 24 hours were not 
Coronary artery disease

Table 2 Univariable and multivariable predictors of obstructive coronary artery disease in patients with unstable angina

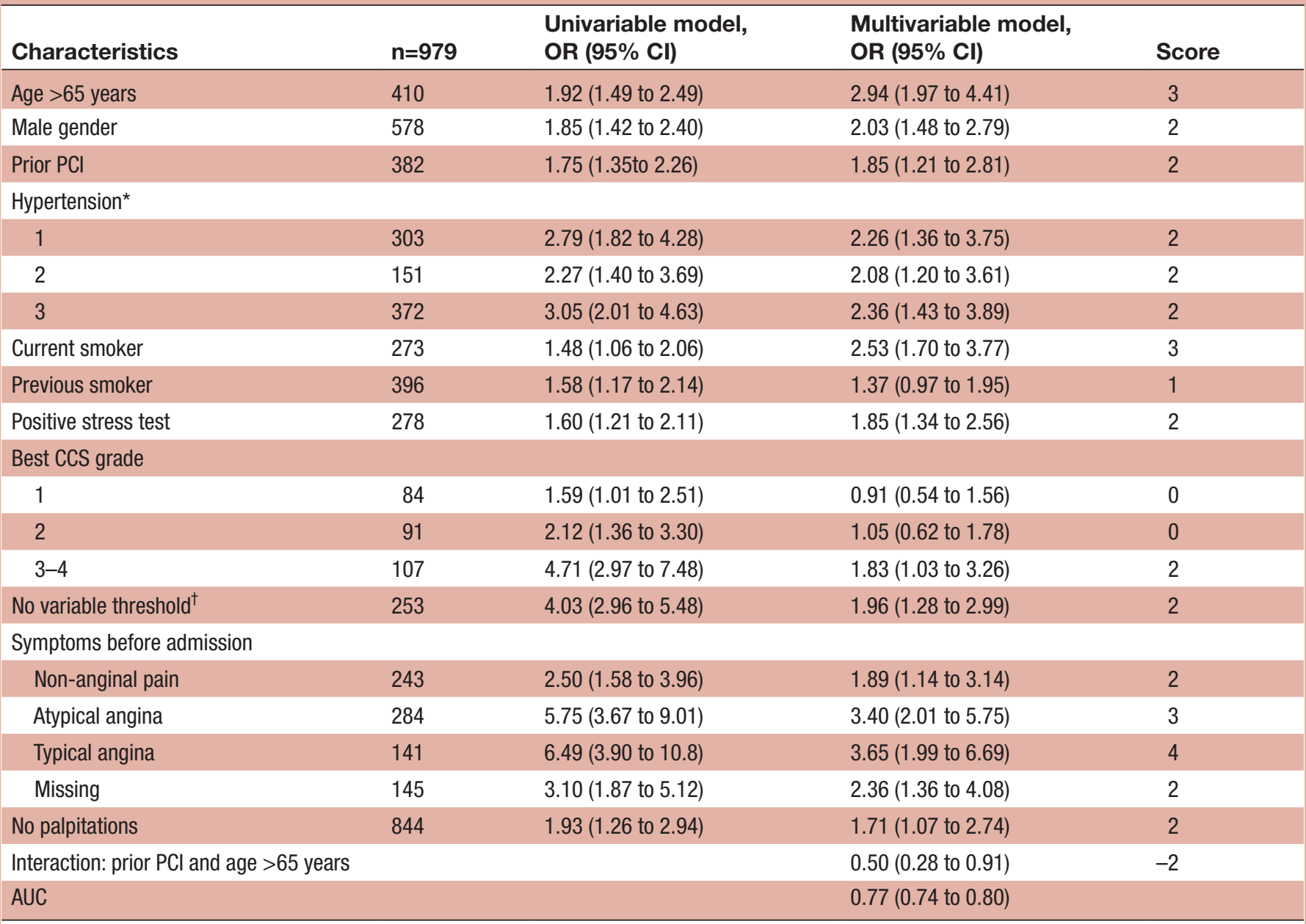

*(1) Use of antihypertensive drugs and normal blood pressure on admission, (2) high blood pressure on admission, (3) 1+2.

†No random variation in the threshold of angina defined by two or more CCS grades.

AUC, area under the curve; CCS, Canadian Cardiovascular Society grading of angina pectoris; PCI, percutaneous intervention .

\begin{tabular}{|c|c|c|c|c|}
\hline Score & $\mathbf{n}$ & $\begin{array}{l}\text { Obstructive CAD, } \\
\text { n (row \%) }\end{array}$ & $\begin{array}{l}\text { Prognostic significant CAD, } \\
\text { n (row \%) }\end{array}$ & $\begin{array}{l}\text { Revascularised, } \\
\text { n (row \%) }\end{array}$ \\
\hline$\leq 5$ & 25 & - & - & - \\
\hline $6-7$ & 54 & $4(7.4 \%)$ & - & $3(5.6 \%)$ \\
\hline 8 & 74 & $15(20 \%)$ & $2(2.7 \%)$ & $15(20 \%)$ \\
\hline 9 & 53 & $12(23 \%)$ & $2(3.8 \%)$ & $12(23 \%)$ \\
\hline 10 & 114 & $26(23 \%)$ & $3(2.6 \%)$ & $23(20 \%)$ \\
\hline 11 & 106 & $42(40 \%)$ & $7(6.6 \%)$ & $35(33 \%)$ \\
\hline 12 & 120 & $49(41 \%)$ & $12(10 \%)$ & $36(30 \%)$ \\
\hline 13 & 101 & $56(55 \%)$ & $17(17 \%)$ & $46(46 \%)$ \\
\hline 14 & 90 & $63(70 \%)$ & $11(12 \%)$ & $51(57 \%)$ \\
\hline 15 & 84 & $49(58 \%)$ & $10(12 \%)$ & $46(55 \%)$ \\
\hline $16-17$ & 97 & $78(80 \%)$ & $23(24 \%)$ & $69(71 \%)$ \\
\hline$\geq 18$ & 61 & 49 (80 \%) & $16(26 \%)$ & $46(75 \%)$ \\
\hline AUC & & $0.77(0.74-0.79)$ & $0.72(0.68-0.77)$ & $0.75(0.71-0.78)$ \\
\hline
\end{tabular}

AUC, area under the curve; CAD, coronary obstructive artery disease. 
associated with obstructive CAD. Neither were chest wall pain, pain related to breathing or self-reported similarity to prior CAD symptoms, but most patient records lacked this information. A GRACE risk score $\geq 109$ was not significantly associated with obstructive CAD (OR 1.37, 95\% CI 0.92 to 2.04 ).

In sensitivity analyses of the 340 patients included after the implementation of hs-cTnT, the AUC of the multivariabel model improved from 0.77 (95\% CI 0.74 to 0.80 ) to 0.81 (95\% CI 0.76 to 0.85 , although with larger CIs) (online supplementary Table 1). The derived risk score performed similarly on the subpopulation with OR of 1.39 (95\% CI 1.28 to 1.52). With a cut-off level of $<13$, we were able to exclude or delay $59 \%$ of the patients $(n=201)$ to acute CAG with an NPV of $96 \%$ for prognostic CAD. Further, with a cut-off level of $<9$ the score demonstrated an NPV of $96 \%$ for any obstructive CAD in $21 \%$ of the patients $(\mathrm{n}=73)$ (online supplementary Table 2$)$.

\section{DISCUSSION}

In our population-based cohort, we have demonstrated that patients with presumed UA referred for acute CAG have low rates of obstructive CAD and low GRACE risk score. By implementing symptom characteristics and clinical information in a new risk score, it was possible to rule out a higher number of patients with lower rates of obstructive CAD than by applying guidelines risk criteria.

There is to our knowledge no other studies using symptoms to predict obstructive CAD in patients with UA. The HEART score includes the clinicians' suspicion of critical disease to predict the risk of MI, PCI, CABG and death in an all-cause chest pain population. ${ }^{16}$ In stable angina, typical angina symptoms added to risk scores is known to improve the prediction of obstructive CAD. ${ }^{17} 18$ In our study, we found that a history of typical angina with a stable or consistently decreasing threshold in the time prior to the acute admission was strongly associated with obstructive $\mathrm{CAD}$. The acute presentation leading to admission was of less importance. Traditional risk factors such as age and smoking were also strongly associated with obstructive $\mathrm{CAD}$, followed by male gender, hypertension and prior PCI. Age however, was significantly reduced as risk factor for those with prior PCI. This led to a positive interaction term in the model and could indicate a protective effect of PCI, secondary prevention or most likely both.

\section{The definition of unstable angina}

Our population underwent clinical decision-making before referral. However, with rates of obstructive CAD as low as $29 \%$ in the end of the study period, patient selection was poor. It seems other aetiologies for chest pain dominated. We found that palpitations, a known symptom of panic disorder, was associated with lack of obstructive CAD on invasive angiography. ${ }^{19}$ Gastrointestinal, musculoskeletal and panic disorders are all known to be highly prevalent in patients with acute or stable chest pain and no evidence of myocardial ischemia, and were likely prevalent in our population. ${ }^{20-22}$

Braunwald and Morrow suggested that increasingly sensitive troponins would make UA a redundant diagnosis. ${ }^{23}$ However in clinical practice, UA remains a challenging diagnosis as objective criteria are rarely present. The fear of uncertainty among clinicians and patients may lead to overuse of presumed UA as indication for acute CAG, even in patients with low clinical suspicion of CAD. This is a likely cause for the low rates of obstructive CAD in our population. Despite the low prevalence of CAD in our population, $79 \%$ satisfied the guidelines criteria for acute CAG within 72 hours, which in our opinion warrants better pre-test selection criteria.

\section{Relevance of guidelines risk criteria and GRACE risk score in the unstable angina population}

The GRACE risk score predicts 3-year mortality in acute coronary syndrome with a superior AUC of $0.82 .^{10}$ The overall low GRACE score observed in both patients with and without obstructive CAD is reassuring and supports a low mortality in the present-day UA population. It may also explain why the GRACE risk score and guidelines encompassing non-ST elevation MI (NSTEMI) had poor discriminative ability for obstructive CAD in patients with UA. ${ }^{310}$ The low mortality of hs-cTn-negative UA is also demonstrated in other studies, with a 90-day mortality and MI rate for hs-cTnT-negative UA patients of $0.6 \%$ and $1.7 \%$, respectively. ${ }^{8}$ Even high-risk UA have a 30 -day combined death and MI rate of approximately $2 \% .{ }^{724}$

Guidelines for NSTEMI/UA recommend an invasive approach in many patients with UA, but the implications of an invasive strategy are not known. Available trials do not report separate findings for patients with or have not implemented hs-cTn assays to discriminate between MI and UA. Meta-analyses of existing trials up to 2015 differ in opinion of the benefit of routine revascularisation for the combined UA and NSTEMI population. ${ }^{25-28}$ It seems likely that hs-cTn-negative UA patients will have less benefit than NSTEMI patients.

The low rate of obstructive CAD, MI and death, as well as an unsure prognostic benefit of revascularisation in the hs-cTn-negative UA patients, questions the resource utilisation of acute CAG in most patients with UA. Our study indicates that it is possible to rule out or delay CAG in more than half of the patients with UA by implementing a new risk score with symptoms characteristics and clinical information in addition to risk criteria in guidelines.

\section{Strengths and limitations}

The major strengths of our study are the inclusion of all consecutive CAGs performed on the indication of UA within a confined geographical area for eight subsequent years, and that all variables included in our risk score are obtained in daily clinical practice. A potential limitation is the relatively small numbers of patients with prognostic significant CAD. We have exclusively investigated patients with UA referred for CAG, and thereby do not know how 
the score performs in an extended chest pain/UA population. The accuracy and consistency of the retrospective information collected from hospital records as well as many missing variables are further limitations. To minimise observer bias, the data collection was blinded for the CAG result. However, if the CAG was soon after followed by coronary artery bypass grafting or gastroscopy, this was visible to the data collector, indicating positive or negative findings of the CAG result, respectively. Since we excluded patients with PCI within 30 days, we could not test the post-MI angina criterion, and the ACC/AHA criterion of PCI within 6 months was only applied for 1-6 months. Further, we did not have enough information on ESC guidelines' recurrence of symptoms to validate its potential role. We used the peak troponin value to define patients with MI. Therefore, we cannot exclude a significant bias due to the exclusion of UA patients with chronic hs-cTn elevation without a significant rise and/ or fall (eg, due to chronic heart failure or severe renal dysfunction). However, as these patients have a higher risk of $\mathrm{CAD}$, the authors believe that these patients usually should be offered CAG on a lower threshold and should be addressed in own focused prospective studies. As the adjustment for standard troponin to hs-cTnT was only applicable for patients with troponin values above the limit of detection, we may have included NSTEMI patients before the implementation of hs-cTnT in 2009 .

\section{CONCLUSIONS}

Our results suggest that by structuring symptom characteristics and clinical variables it may be possible to postpone or cancel acute CAG in over half of the patients referred with presumed UA. This would reduce cost for healthcare systems, avoid exposing patients to unnecessary risk of complications and release capacity for more critical diagnoses. Prospective studies are needed to validate our findings.

Acknowledgements The authors extend gratitude to all invasive cardiologist involved in the clinical registry of coronary angiography at the University Hospital of North Norway.

Contributors KF collected and analysed the data, interpreted the results and drafted the manuscript. AK interpreted the results and drafted the manuscript. JTM interpreted the results and critically reviewed the manuscript. TT organised the data collection, interpreted the results and critically reviewed the manuscript. HS organised the data collection, collected and analysed the data, interpreted the results and drafted the manuscript.

Funding Research student grant from UiT The Arctic University of Norway, Troms $\emptyset$. Competing interests None declared.

Patient consent Not required.

Ethics approval The study was a clinical audit and therefore not subject to evaluation by the Regional Committee of Ethics. It was approved by the Data Protection Official for Research at the University Hospital of North Norway (\#0217).

Provenance and peer review Not commissioned; externally peer reviewed.

Data sharing statement No additional data are available.

Open access This is an open access article distributed in accordance with the Creative Commons Attribution Non Commercial (CC BY-NC 4.0) license, which permits others to distribute, remix, adapt, build upon this work non-commercially, and license their derivative works on different terms, provided the original work is properly cited, appropriate credit is given, any changes made indicated, and the use is non-commercial. See: http://creativecommons.org/licenses/by-nc/4.0/.

\section{REFERENCES}

1. Niska R, Bhuiya F, Xu J. National hospital ambulatory medical care survey: 2007 emergency department summary. Natl Health Stat Report 2010;26:1-31.

2. Thygesen K, Alpert JS, Jaffe AS, et al. Third universal definition of myocardial infarction. Nat Rev Cardiol 2012;9:620-33.

3. Roffi M, Patrono C, Collet JP, et al. 2015 ESC Guidelines for the management of acute coronary syndromes in patients presenting without persistent ST-segment elevation: task force for the management of acute coronary syndromes in patients presenting without persistent ST-Segment elevation of the European Society of Cardiology (ESC). Eur Heart J 2016;37:267-315.

4. Reichlin T, Twerenbold R, Reiter M, et al. Introduction of highsensitivity troponin assays: impact on myocardial infarction incidence and prognosis. Am J Med 2012;125:1205-13.

5. Melki D, Lugnegård J, Alfredsson J, et al. Implications of introducing high-sensitivity cardiac troponin $\mathrm{T}$ into clinical practice: data from the SWEDEHEART Registry. J Am Coll Cardiol 2015;65:1655-64.

6. Eggers KM, Lindahl B, Melki D, et al. Consequences of implementing a cardiac troponin assay with improved sensitivity at swedish coronary care units: an analysis from the SWEDEHEART registry. Eur Heart J 2016;37:2417-24.

7. Lindahl B, Venge P, James $S$. The new high-sensitivity cardiac troponin T assay improves risk assessment in acute coronary syndromes. Am Heart J 2010;160:224-9.

8. Vafaie M, Slagman A, Möckel M, et al. Prognostic value of undetectable hs troponin $\mathrm{T}$ in suspected acute coronary syndrome. Am J Med 2016;129:274-82.

9. Amsterdam EA, Wenger NK, Brindis RG. 2014 AHA/ACC Guideline for the management of patients with non-ST-elevation acute coronary syndromes: a report of the American college of cardiology/ American heart association task force on practice guidelines. J Am Coll Cardiol 2014;64:139-228.

10. Fox KA, Fitzgerald G, Puymirat E, et al. Should patients with acute coronary disease be stratified for management according to their risk? Derivation, external validation and outcomes using the updated GRACE risk score. BMJ Open 2014;4:e004425.

11. Omland T, de Lemos JA, Sabatine MS, et al. A sensitive cardiac troponin T assay in stable coronary artery disease. N Engl J Med 2009;361:2538-47.

12. Giannitsis E, Kurz K, Hallermayer K, et al. Analytical validation of a high-sensitivity cardiac troponin T assay. Clin Chem 2010;56:254-61.

13. Task Force Members, Montalescot G, Sechtem U, et al. 2013 ESC guidelines on the management of stable coronary artery disease: the Task Force on the management of stable coronary artery disease of the European society of cardiology. Eur Heart J 2013;34:2949-3003.

14. Kolh P, Windecker S, Alfonso F. 2014 ESC/EACTS Guidelines on myocardial revascularization: the task force on myocardial revascularization of the European Society of Cardiology (ESC) and the European Association for Cardio-Thoracic Surgery (EACTS). Developed with the special contribution of the European Association of Percutaneous Cardiovascular Interventions (EAPCI). Eur $J$ Cardiothorac Surg 20142014;46:517-92.

15. Yusuf S, Zucker D, Peduzzi P, et al. Effect of coronary artery bypass graft surgery on survival: overview of 10-year results from randomised trials by the coronary artery bypass graft surgery trialists collaboration. Lancet 1994;344:563-70.

16. Six AJ, Backus BE, Kelder JC. Chest pain in the emergency room: value of the HEART score. Neth Heart J 2008;16:191-6.

17. Genders TS, Steyerberg EW, Alkadhi H, et al. A clinical prediction rule for the diagnosis of coronary artery disease: validation, updating, and extension. Eur Heart J 2011;32:1316-30.

18. Patel MR, Peterson ED, Dai D, et al. Low diagnostic yield of elective coronary angiography. N Engl J Med 2010;362:886-95.

19. Barsky AJ, Cleary PD, Sarnie MK, et al. Panic disorder, palpitations, and the awareness of cardiac activity. J Nerv Ment Dis 1994;182:63-71.

20. Dammen T, Arnesen H, Ekeberg O, et al. Panic disorder in chest pain patients referred for cardiological outpatient investigation. J Intern Med 1999;245:497-507.

21. Karlson BW, Herlitz J, Pettersson P, et al. Patients admitted to the emergency room with symptoms indicative of acute myocardial infarction. J Intern Med 1991;230:251-8.

22. Katz PO, Dalton CB, Richter JE, et al. Esophageal testing of patients with noncardiac chest pain or dysphagia. Results of three years' experience with 1161 patients. Ann Intern Med 1987;106:593-7. 
23. Braunwald E, Morrow DA. Unstable angina: is it time for a requiem? Circulation 2013;127:2452-7.

24. Grinstein J, Bonaca MP, Jarolim P, et al. Prognostic implications of low level cardiac troponin elevation using high-sensitivity cardiac troponin T. Clin Cardiol 2015;38:230-5.

25. Fanning JP, Nyong J, Scott IA, et al. Routine invasive strategies versus selective invasive strategies for unstable angina and non-ST elevation myocardial infarction in the stent era. Cochrane Database Syst Rev 2016;5:Cd004815.

26. Elgendy IY, Mahmoud AN, Wen X, et al. Meta-analysis of randomized trials of long-term all-cause mortality in patients with non-ST-elevation acute coronary syndrome managed with routine invasive versus selective invasive strategies. Am J Cardiol 2017;119:560-4.

27. O'Donoghue M, Boden WE, Braunwald E, et al. Early invasive vs conservative treatment strategies in women and men with unstable angina and non-ST-segment elevation myocardial infarction: a metaanalysis. JAMA 2008;300:71-80.

28. Fox KA, Clayton TC, Damman P, et al. Long-term outcome of a routine versus selective invasive strategy in patients with non-STsegment elevation acute coronary syndrome a meta-analysis of individual patient data. J Am Coll Cardiol 2010;55:2435-45. 